\title{
Conservadurismo contable, concentración de propiedad y estructuras piramidales: el caso de Chile
}

\author{
Cristian Medina Medina \\ Facultad de Economía y Negocios \\ Universidad de Chile \\ cmedinam@fen.uchile.cl
}

\begin{abstract}
The main objective of this document is to contribute through empirical evidence, to the literature that deals with the concept of accounting conservatism. Specifically, it analyzes how conservative the Chilean financial market is, and analyze the effects that the ownership structure of the firm has on the level of accounting conservatism. The sample used consists of an unbalanced panel with financial information of 119 companies listed on the Santiago de Chile stock exchange for the 2007-2011 study period, obtained from the Thomson One database (Thomson Reuters) and with data collection of the Superintendency of Securities and Insurance (SVS). The results indicate that effectively there is evidence in the Chilean market, that the concentration of property negatively affects the level of accounting conservatism. However, it is not possible to determine that in companies with pyramid-owned structures the level of accounting conservatism decreases.
\end{abstract}

Keywords: Accounting conservatism, Concentration of Property, Pyramidal Structure, Asymmetries of Information, Asymmetrical Recognition of Utilities. 


\section{Resumen}

Este documento tiene como principal objetivo contribuir, mediante evidencia empírica, a la literatura que aborda el concepto del conservadurismo contable. Específicamente se analiza qué tan conservador es el mercado financiero chileno y analizar los efectos que tiene la estructura de propiedad de las empresas en el nivel de conservadurismo contable. La muestra utilizada consiste en un panel no balanceado con información financiera de 119 empresas cotizadas en la Bolsa de Santiago de Chile para el periodo de estudio 2007-2011, obtenida desde la base de datos de Thomson One (Thomson Reuters) y con recolección de datos de la Superintendencia de Valores y Seguros (SVS). Los resultados indican que efectivamente en el mercado chileno se evidencia que la concentración de propiedad influye negativamente en el nivel de conservadurismo contable. No obstante, no es posible determinar que en empresas con estructuras de propiedad piramidales disminuya el nivel de conservadurismo contable.

Palabaras clave: Conservadurismo contable, concentración de propiedad, estructura piramidal, asimetrías de información, reconocimiento asimétrico de las utilidades.

\section{Introducción}

Debido a los distintos escándalos y problemas financieros que han ocurrido en las últimas décadas, es que se hace cada vez más necesario no solo contar con mercados financieros eficientes, sino que también conocer su comportamiento, para de este modo obtener información financiera oportuna $\mathrm{y}$ transparente para los inversionistas y para el mercado en general. De hecho, las autoridades reguladoras han tomado conciencia acerca del rol que cumple la información financiera y han promulgado reformas a los sistemas financieros, incluyendo aquellas relacionadas con el gobierno corporativo, las normativas contables y con el papel que 
cumple la función de auditoría (Klapper y Love, 2004; Kothari et al., 2010; Baxter y Cotter, 2009).

En este sentido, los estados financieros, y en especial las utilidades contables, juegan un papel fundamental como fuente de información transparente, siendo un factor clave en el proceso de toma de decisiones. El grado de conservadurismo de las utilidades contables, entendida como la tendencia a reconocer asimétricamente las buenas y malas noticias económicas en las utilidades contables con el tiempo (Givoly et al., 2007; Roychowdhury y Watts, 2007), puede ser un factor relevante al momento de velar por información oportuna y fidedigna, mitigando ciertos problemas de información asimétrica (Watts, 2003a; Watts, 2003b).

Sin embargo, también es necesario entender cómo se comporta este conservadurismo contable, analizando en detalle cuáles son los factores por los que se ve afectado. De hecho, existen diversos estudios respecto de conservadurismo contable, entre ellos se señala que la concentración de propiedad aumenta la probabilidad de que los problemas de información asimétrica entre los gerentes y los accionistas se resuelva mediante canales de comunicación privados en vez de recurrir a información pública, reduciendo la demanda por conservadurismo (Peek et al., 2010; Ball y Shivakumar, 2006; La Fond, 2005; Dargenidou et al., 2007). Esto último es consistente con La Fond (2005) y con Villalonga y Amit (2006), quienes señalan que la concentración de propiedad ayuda a los propietarios a economizar los costos del monitoreo de los gerentes, disminuyendo los problemas de agencia.

Este documento tiene como principal objetivo contribuir a este tipo de literatura, aportando sustento empírico mediante la comprobación de hipótesis. Específicamente, se analiza qué tan conservador es el mercado financiero chileno y analizar los efectos que tiene la estructura de propiedad de las empresas en el nivel de conservadurismo contable.

Este estudio se estructura de la siguiente forma: partiendo con un repaso de la literatura referente al conservadurismo contable 
y el efecto que tiene en él la concentración de propiedad y las estructuras de las empresas, donde también se dejan establecidas las hipótesis que se desean contrastar empíricamente. A continuación se hace una descripción de la muestra objeto de estudio, así como la presentación de las variables y metodología empleada para el análisis, después se expondrán los resultados obtenidos y comentaremos algunas de sus implicaciones. La investigación finaliza con un apartado en el que se exponen las conclusiones alcanzadas.

\section{Marco teórico}

El conservadurismo de las utilidades contables se entiende como la tendencia a reconocer asimétricamente las buenas y malas noticias económicas en las utilidades contables con el tiempo (Roychowdhury y Watts, 2007), es decir, una empresa se define como conservadora si al momento de enfrentarse a malas noticias, estas son reconocidas inmediatamente en la contabilidad de la empresa, por el contrario, cuando se enfrenta a buenas noticias, estas no se reflejan de inmediato en la contabilidad, debido a que necesitan un mayor nivel de confirmación de esta información. Este reconocimiento asimétrico de las noticias puede ser un factor relevante al momento de velar por tener información oportuna y fidedigna, combatiendo ciertos problemas de agencia (Watts, 2003a; Watts, 2003b).

Técnicamente, el conservadurismo contable es el reconocimiento asimétrico de las pérdidas versus las ganancias de los flujos de caja futuros esperados, es decir, es reconocer aceleradamente las pérdidas económicas en los reportes financieros en vez de las ganancias. Esta práctica contable reduce los problemas de agencia asociados con las decisiones de inversión de los gerentes (insiders), ya que estos saben que las pérdidas serán reconocidas inmediatamente en sus mandatos, mientras que las ganancias 
probablemente serán reconocidas en gerencias posteriores, por esta razón solo tomarán proyectos que sean rentables (Ball, 2001).

Mientras un mercado informado reconoce los efectos económicos de los eventos tan pronto como ellos ocurren, la contabilidad puede no reflejar tal información inmediatamente. El reconocimiento asimétrico de la información contable de las ganancias, o conservadurismo contable, captura la tendencia a requerir un alto grado de confirmación de la información para reflejar las buenas noticias como ganancias y un menor grado de confirmación para reconocer las malas noticias como pérdidas (Basu, 1997).

El conservadurismo contable permite a los inversores externos anticipar las consecuencias negativas de las acciones tomadas por los insiders y reduce la probabilidad de que estos obtengan beneficios privados mediante inversiones que no maximicen valor -por ejemplo iniciando proyectos con un VAN negativo, o manteniendo proyectos que han generado pérdidas (Ball, 2001)-, debido a que el hecho de que estos sepan que los proyectos malos serán reconocidos oportunamente creará incentivos ex ante para que los managers rechacen proyectos malos (Bushman et al., 2006). Por tanto, el conservadurismo contable mitiga los problemas de agencia asociados con las decisiones de inversiones de los gerentes al exigir reportes de las pérdidas en el momento que las expectativas no son buenas (Ball y Shivakumar, 2006).

Sin embargo, no todas las empresas tienen la misma probabilidad de usar el conservadurismo contable. En La Fond y Roychowdhury (2008) se muestra que empresas que tienen una alta concentración de propiedad reportan menos conservadurismo contable, debido a que los accionistas no tienen incentivos a monitorear a los gerentes, ya que hacerlo sería muy difícil y costoso (Jensen y Meckling, 1976). Esto es consistente con que los accionistas demandan un gran nivel de conservadurismo como una manera de abordar los problemas de agencia que vienen de la separación entre el control y la propiedad. Si los accionistas tienen 
gran parte de su riqueza en la empresa, ellos tendrán enormes incentivos a monitorear la empresa constantemente. Por tanto, la concentración de la propiedad puede reducir los problemas de agencia entre los accionistas y los gerentes, reduciendo la demanda por conservadurismo contable. Por otra parte, los propietarios controladores son más propensos a confiar en los canales de comunicación privados (en vez de ocupar información pública) como una manera de reducir los problemas de asimetrías de información entre accionistas y los gerentes, y que también disminuye la demanda por conservadurismo contable (Peek et al., 2010).

Bona et al. (2011) investigan si esas diferencias específicas de las empresas pueden explicar las diferencias en los niveles observados de conservadurismo contable, y también encuentran que a medida que aumenta la participación en la propiedad del accionista controlador disminuye la demanda por conservadurismo contable.

Por otra parte, existen empresas con la presencia de un accionista controlador pero con diferentes niveles de propiedad, es decir, presenta diferencias entre los derechos de voto y derechos de flujos de caja (La Porta et al.,1999). Este tipo de diferencias entre el control y la propiedad del accionista controlador es característica de empresas con estructuras de propiedad piramidales. En ellas, el problema de información asimétrica entre los accionistas y los gerentes tiene más probabilidades de ser mitigada, mientras que la asimetría de información existente entre los insiders (gerentes de la empresa y accionistas controladores) y los outsiders (accionistas minoritarios de la empresa) es probable que sea mayor.

Entonces, en este documento se busca aportar a este tipo de literatura que trata acerca de los efectos que tiene la estructura de propiedad en el conservadurismo contable, analizando si las empresas que tienen un controlador mayoritario abordan el problema de agencia de información asimétrica usando el reconocimiento inmediato de las pérdidas, depende: 
- Del grado de participación en la propiedad del accionista controlador

- A diferencia entre los derechos de voto y los derechos de flujo de caja del accionista controlador.

\section{A. Desarrollo de hipótesis}

\section{a. CONCENTRACIÓN DE PROPIEDAD}

De acuerdo con La Fond (2005), a medida que la propiedad del controlador aumenta, también aumentan los incentivos a compartir información con el propietario dominante debido a la mayor parte del capital que es suministrado por ese accionista. A su vez, los problemas de asimetría de información entre los gerentes y los accionistas probablemente se resolverá mediante canales de comunicación privados que se desarrollan mediante relaciones informales (Ball y Shivakumar, 2006), reduciendo la demanda por conservadurismo contable como forma de monitoreo. Con esto, se predice una relación negativa entre la participación de propiedad del accionista controlador y el conservadurismo contable.

Watts (2003a, 2003b) propone una explicación alternativa para la influencia que tiene la estructura de propiedad en el conservadurismo contable, en la que se podría decir que a medida que aumenta la participación en la propiedad del controlador, probablemente la empresa tendrá menos canales externos de comunicación, debido a la pequeña proporción de capital que aportan los accionistas minoritarios. Esta reducida cantidad de canales externos de estos accionistas que no tienen acceso a la información privada de la compañía reducirá la demanda por conservadurismo contable. Asimismo, a medida que aumenta la propiedad del accionista controlador, la empresa hará menos contrataciones externas (Watts, 2003a, 2003b; La Fond, 2005), debido a una relativamente menor porción de capital que tienen los 
accionistas minoritarios. La reducción en la necesidad de las empresas por mercados de capitales externos para recaudar fondos reduce la demanda de conservadurismo contable como forma de mitigar los problemas de asimetrías de información entre insiders y los accionistas minoritarios. Entonces, el rol externo del conservadurismo contable también predice una relación negativa entre la participación de la propiedad del accionista controlador y el conservadurismo contable.

De este modo, tanto por el rol que tiene el monitoreo y la contratación externa sobre el conservadurismo contable, se predice una relación negativa entre la participación en la propiedad del accionista controlador y el conservadurismo contable. Más formalmente:

Hipótesis 1: "Mientras mayor sea la participación en la propiedad del dueño mayoritario, menor es el nivel de conservadurismo contable".

\section{b. ESTRUCTURA PIRAMIDAL}

En cuanto a la diferencia entre los derechos de voto y derechos de flujos de caja, Bebchuk (1999) argumenta que esta separación tiende a ser asociada con estructuras de concentración de propiedad piramidales. Argumento que encuentra sustento en Francis et al. (2005), quienes observan que las estructuras de propiedad que separan los derechos de voto de los derechos de flujos de caja son comunes fuera de EE.UU.; y en La Porta et al. (1999), quienes muestran que esta estructura de propiedad es común en países en que su sistema legal ofrece menos protección a los intereses de los accionistas minoritarios. Estas estructuras piramidales resultan del hecho en que el accionista mayoritario tiene directo control de una empresa, y esta a su vez controla otra empresa, que puede controlar en sí mismo a otra empresa, etcétera (Almeida y Wolfenzon, 2006). 
Mediante la separación del control de propiedad, estas estructuras piramidales también permiten al propietario controlador crear un mercado de capital interno que puede ser usado para financiar proyectos (Cestone y Fumagalli, 2005). De hecho, Desai et al. (2004) encuentran que este tipo de mercados de capitales internos tienden a ser empleados para obtener fondos a un costo más bajo que en el mercado de capitales externo. De acuerdo con esto, cuando aumenta la diferencia entre los derechos de voto y los derechos de flujo de caja del propietario controlador, los accionistas minoritarios y el mercado, razonablemente van a creer que el accionista controlador puede expropiar o actuar en contra de los accionistas minoritarios, por lo que se esperaría que la empresa se enfrente a mayores costos de contratación externa (Faccio et al., 2001). Este aumento de los costos de contratación externa probablemente hace que la empresa recurra al mercado de capitales interno, creado mediante su estructura piramidal, para financiar el crecimiento de la compañía. La disminución del uso de contratos externos disminuye la demanda por conservadurismo contable como una forma de mitigar la información asimétrica entre los insiders y los accionistas minoritarios. Por tanto, el rol de los contratos externos del conservadurismo contable también predice una relación negativa entre la diferencia de los derechos de voto y derechos en el flujo de caja y el conservadurismo contable.

Además, un aumento en la diferencia entre los derechos de voto y los de flujo de caja aumenta las preocupaciones del accionista controlador sobre la dilución de su dominio de acciones. Esta preocupación puede ser especialmente relevante cuando el accionista mayoritario tiene bajos derechos de flujo de caja, por lo que puede ser fácilmente superado en la participación accionaria por un nuevo accionista (Du y Dai, 2005). Para evitar este efecto de dilución, el accionista controlador probablemente también dependerá más de los mercados de capital internos para financiar proyectos. El mayor uso del mercado de capital interno incrementa los incentivos de los gerentes a compartir información con el 
accionista controlador. Por tanto, el rol de monitoreo del conservadurismo contable predice una relación negativa entre la diferencia de derechos de voto y derechos de flujo de caja del propietario controlador y el conservadurismo contable.

Por último, esta preferencia por los mercados de capitales internos aumenta los incentivos de los gerentes para compartir información con el accionista controlador, por lo que la asimetría de información entre el gerente y los accionistas probablemente se resolverá por canales de comunicación privados.

Por tanto, en conjunto, estos argumentos sugieren una relación negativa entre la diferencia entre los derechos de voto y de flujo de caja del accionista mayoritario y el conservadurismo contable. Más formalmente:

Hipótesis 2: "Mientras mayor sea la diferencia entre los derechos de voto y los derechos de flujos de caja del accionista controlador, menor es el nivel de conservadurismo contable".

\section{Muestra, modelos y variables}

\section{A. $\quad$ Muestra}

La data utilizada consiste en un panel no balanceado de 529 observaciones y 119 empresas cotizadas en la Bolsa de Santiago de Chile para el periodo de estudio 2007-2011. La información para el análisis empírico se obtuvo por medio de distintas fuentes de información. En primer lugar se ha empleado Thomson One (Thomson Reuters) con la finalidad de obtener información financiera confiable y alguna información de la propiedad de las empresas. Luego, para identificar el último accionista y estimar la existencia de separación entre derechos de voto y derechos de flujo de caja se ha recurrido a un proceso de recolección manual de datos, 
que incluye información descargada de la Superintendencia de Valores y Seguros (SVS), memorias anuales, noticias de prensa, información corporativa de las correspondientes páginas webs, así como también búsqueda de escrituras de constituciones de sociedades que participan en la propiedad de las empresas pero que no son reguladas por entidades como la SVS. Esta metodología identifica al último propietario de la empresa para cada año, lo que ayuda a mitigar los problemas asociados con asumir que la estructura de propiedad de la empresa se mantiene sin cambios durante el periodo de la muestra.

Como se mencionó anteriormente, las estructuras piramidales son la forma más común de separar los derechos de voto de los derechos de flujo de caja en el caso chileno. Por tanto, considerando toda la literatura que trata acerca de la existencia de un último accionista (La Porta et al., 1999; Claessens et al., 2000; Faccio y Lang, 2002), se ha seguido varios pasos para identificar el último accionista de la cadena de control $\mathrm{y}$, específicamente, la concentración de la propiedad, la participación en los derechos de voto, derechos por sobre los flujos de caja de la empresa y el grado de separación entre ambas.

La metodología comienza por la identificación de la cadena de control de la empresa hasta llegar al último propietario. Esto es, identificar al principal accionista o empresa controladora (o ambas si hay participación directa e indirecta) de la empresa más alejada de la cadena de propiedad, para luego analizar el principal accionista o empresa de la organización que se encuentra un eslabón más arriba de la cadena de propiedad, y así sucesivamente hasta llegar al último accionista quien controla toda la cadena de propiedad. Es admisible señalar que esta metodología típicamente asume que el accionista debe tener al menos $20 \%$ de los votos totales (directa e indirectamente), suficiente para que un accionista se considere el controlador de una compañía.

Una vez identificada la identidad del último accionista, se procede a calcular los derechos de voto y derechos de flujo de caja. 
Para el cálculo de los derechos de voto se considera que cuando existen múltiples accionistas en la cadena de control que poseen más del $10 \%$ de la propiedad, los derechos de voto del último accionista se obtienen como el porcentaje de la propiedad que el último accionista logra de manera directa e indirecta, y en aquellos casos donde existen participaciones indirectas se considera el "vínculo más débil" de la cadena o la menor participación dentro de la cadena.

A continuación se procede a generalizar el cálculo de los derechos de voto (DV), los derechos de flujo de caja (DFC), y la separación (DVDFC) del último accionista. Primero, los derechos de voto (DV) del último accionista de la cadena se obtienen como:

$$
D V=\operatorname{Min}_{\{1, \ldots, n\}}\left\{\alpha_{i}\right\}
$$

Donde $\alpha_{\mathrm{i}}$ corresponde a los derechos de voto de cada empresa $i$, a lo largo de una cadena de control que va desde 1 hasta $n$ empresas, hasta llegar al último propietario. Por otro lado, los derechos de flujos de caja (DFC) del último accionista de la cadena se obtienen calculando lo siguiente:

$$
D F C=\prod_{i=1}^{n} D V
$$

Es decir, estos derechos corresponden a la multiplicación de todos los derechos de voto desde la empresa $i=1$ hasta $n$ empresas que conforman la cadena de control hasta llegar al último propietario.

Para ilustrar lo anterior, supongamos que, por ejemplo, si el "Accionista 1" es el accionista principal de la "Empresa A", con un $26 \%$ de sus derechos de voto, y a su vez la "Empresa A" es el accionista principal de la "Empresa B", con 32\% de sus derechos de voto, entonces podemos decir que la "Empresa B" es controlada por el "Accionista 1", con un nivel de control del 26\% (min $(26 \%$; $32 \%)$ ). En este caso, el último propietario de la "Empresa B" 
controla la compañía indirectamente por medio de la "Empresa A". Tenga en cuenta, sin embargo, que el "Accionistas 1" solo tiene el 8,32\% de los derechos de flujo de caja de la "Empresa B", que viene dada por el producto de los porcentajes de tenencias de los accionistas a lo largo de la cadena $(0,0832=0,26 * 0,32)$. La utilización de esta estructura necesariamente implica que exista una diferencia entre los derechos de voto y los derechos de flujos de caja del accionista controlador. Esta distinción es importante, ya que, mientras que los derechos de flujos de caja miden los derechos económicos de los accionistas, los derechos de voto miden el control que tienen los accionistas respecto de las decisiones más importantes de la compañía, como decisiones relativas a las fusiones, absorciones, elección de directores o la elección de los altos gerentes.

Para calcular esta diferencia, propia de las estructuras piramidales, entre los derechos de voto (control) y los derechos de flujo de caja (propiedad) del último propietario (DVDFC) se tienen el siguiente cálculo:

$$
D V D F C=D V-D F C
$$

Para las empresas que no presentan separación, la separación será igual a 0 , y para las empresas con derechos de voto superiores a los de flujo de caja esta medida será mayor que 0 .

\section{B. Modelos y variables}

Siguiendo trabajos previos, se utilizó el modelo propuesto por Basu (1997), que mide el reconocimiento asimétrico de las utilidades como medida de conservadurismo contable. Este modelo utiliza las rentabilidades anuales de las acciones como medida proxy de las buenas y malas noticias y que se sustenta en la intuición de que los precios de las acciones incorporan oportunamente toda la 
información que arriba al mercado desde las múltiples fuentes informativas (incluyendo las utilidades reportadas), por lo que los cambios en los precios son una medida que captura todas las noticias durante un periodo. En concreto, Basu (1997) propone un modelo en el que los ingresos (contables) dependen de los retornos (positivos y negativos), como proxy de las buenas y malas noticias:

Ecuación (1):

$$
X_{i t}=\alpha+\beta_{1} * R_{i t}+\beta_{2} * D R_{i t}+\beta_{3} * D R_{i t} * R_{i t}
$$

Donde $X$ representa las utilidades por acción dividida por el precio de la acción al comienzo del periodo; $R$ representa la rentabilidad anual de los precios de las acciones y $D R_{i t}$ es una variable dummy que toma el valor 1 cuando la rentabilidad anual de los precios de las acciones $\left(R_{i t}\right)$ es negativa, y cero cuando es positiva. Por tanto, la respuesta de las ganancias a las malas noticias es capturada por el término $D R_{i t} * R_{i t}$.

El coeficiente $\beta_{1}$ mide la respuesta de las ganancias contables a las buenas noticias, mientras que la sensibilidad a las malas noticias es capturada por la combinación de $\beta_{1}$ y $\beta_{3}$. El coeficiente $\beta_{3}$ captura la sensibilidad de las utilidades a las malas noticias o, en otras palabras, el nivel de reconocimiento asimétrico de las utilidades (o conservadurismo) y es de esperar que este sea positivo y significativo, comúnmente llamado coeficiente de asimetría.

Con el fin de analizar la influencia que tiene el nivel de participación en la propiedad del accionista mayoritario final, se realiza una extensión del modelo propuesto por Basu (1997) para incluir una variable del nivel de los derechos de flujo de caja que tiene el dueño final de la empresa $(D F C)$, por lo que se tiene la siguiente ecuación: 
Ecuación (2):

$$
\begin{aligned}
X_{i t}= & \alpha+\beta_{1} * R_{i t}+\beta_{2} * D R_{i t}+\beta_{3} * D R_{i t} * R_{i t}+\beta_{4} * D F C_{i t} \\
& +\beta_{5} * R_{i t} * D F C_{i t}+\beta_{6} * D R_{i t} * D F C_{i t}+\beta_{7} * D R_{i t} * R * D F C_{i t} .
\end{aligned}
$$

También se realiza otra extensión al modelo de Basu (1997) para analizar la influencia que tiene el nivel de participación en la propiedad del accionista mayoritario final, pero esta vez desde el punto de vista del grado de derechos de voto que tiene el dueño final de la empresa $\left(D V_{i t}\right)$, por lo que se tiene la siguiente ecuación:

Ecuación (3):

$$
\begin{aligned}
X_{i t}= & \alpha+\beta_{1} * R_{i t}+\beta_{2} * D R_{i t}+\beta_{3} * D R_{i t} * R_{i t}+\beta_{4} * D V_{i t} \\
& +\beta_{5} * R_{i t} * D V_{i t}+\beta_{6} * D R_{i t} * D V_{i t}+\beta_{7} * D R_{i t} * R * D V_{i t} .
\end{aligned}
$$

En las ecuaciones (2) y (3), al igual que en la ecuación original de Basu (1997), la sensibilidad de las ganancias a una mala noticia para las empresas sigue siendo capturada por los coeficientes $\beta_{1}$ y $\beta_{3}$. Por otra parte, $\beta_{5} \mathrm{y} \beta_{7}$ capturan el efecto del aumento del grado de propiedad que tiene el accionista mayoritario. Por tanto $\beta_{3}$ entrega el coeficiente de asimetría marginal que tienen las malas noticias dependientes del nivel de propiedad que tiene el accionista mayoritario, y $\beta_{7}$ estima la asimetría marginal del nivel de propiedad del accionista mayoritario.

Por último, para evaluar la segunda hipótesis que se planteó, se realiza otra extensión al modelo de Basu (1997), con el fin de analizar el comportamiento que tiene el conservadurismo contable, si al efecto de la propiedad se añade el hecho de tener estructuras piramidales de control. Para ello se agrega una variable dummy que toma el valor 1 cuando existe diferencia entre el derecho de voto y derecho de flujo de caja que tiene el accionista mayoritario, y 0 en caso contrario: 
Ecuación (4):

$$
\begin{aligned}
X_{i t}= & \alpha+\beta_{1} * R_{i t}+\beta_{2} * D R_{i t}+\beta_{3} * D R_{i t} * R_{i t}+\beta_{4} * D F C_{i t} \\
& +\beta_{5} * R_{i t} * D F C_{i t}+\beta_{6} * D R_{i t} * D F C_{i t}+\beta_{7} * D R_{i t} * R * D F C_{i t} \\
& +\beta_{8} * D V D F C_{i t}+\beta_{9} * R * D V D F C_{i t}+\beta_{10} * D R_{i t} * D V D F C_{i t} \\
& +\beta_{11} * D R_{i t} * R_{i t} * D V D F C_{i t} .
\end{aligned}
$$

Donde $\beta_{3}$ entrega el coeficiente de asimetría marginal que tienen las malas noticias dependientes del nivel de propiedad que tiene el accionista mayoritario y de la existencia de separación entre los derechos de voto y derechos de flujos de caja del accionista mayoritario, $\beta_{7}$ estima el coeficiente de asimetría marginal del nivel de propiedad del accionista mayoritario, y $\beta_{11}$ estima el coeficiente de asimetría marginal que aporta la existencia de separación de propiedad entre los derechos de voto y derechos de flujos de caja del accionista controlador.

En todas las ecuaciones antes mencionadas, para controlar la incidencia de otras variables que podrían afectar al conservadurismo contable, se incluyen ciertas variables de control: apalancamiento (Lev), medido como deuda total dividida por el total de activos al cierre del ejercicio; la variable size, medida como el logaritmo natural del valor de mercado del patrimonio; y variables de control temporales (año) y de corte transversal (sector económico). Además se incluye una variable dummy que indica si la empresa cuenta o no con IFRS, esto último basado en el trabajo de Jara y Arias (2012), quienes encuentran que el cambio de normativa contable desde NIC a IFRS que ocurrió en Chile en 2009 generó un aumento del grado de conservadurismo contable que presentan las empresas chilenas.

\section{Resultados}

En la tabla 1 se muestran los resultados de las estimaciones para la ecuación (2), utilizando distintas interacciones de las variables de control, en donde se analiza el efecto de la concentración de 
propiedad que tiene el accionista mayoritario de la empresa respecto del conservadurismo contable.

\section{Tabla 1}

\section{Conservadurismo contable y derechos de flujo de caja}

\begin{tabular}{|c|c|c|c|c|c|c|}
\hline & (1) & (2) & (3) & (4) & (5) & (6) \\
\hline VARIABLES & $\mathrm{X}$ & $\mathrm{X}$ & $\mathrm{X}$ & X & $\mathrm{X}$ & $\mathrm{X}$ \\
\hline \multirow[t]{2}{*}{$\bar{R}$} & 0,001 & 0,001 & $-0,000$ & $-0,005$ & $-0,004$ & $-0,012$ \\
\hline & $(0,059)$ & $(0,059)$ & $(0,059)$ & $(0,058)$ & $(0,058)$ & $(0,058)$ \\
\hline \multirow[t]{2}{*}{ DR } & $-0,072 *$ & $-0,073 *$ & $-0,066$ & $-0,080^{*}$ & $-0,075^{*}$ & $-0,075^{*}$ \\
\hline & $(0,041)$ & $(0,041)$ & $(0,041)$ & $(0,041)$ & $(0,041)$ & $(0,041)$ \\
\hline \multirow[t]{2}{*}{ DRxR } & $0,201^{*}$ & $0,205^{*}$ & $0,202 *$ & $0,201 *$ & $0,252 * *$ & $0,253 * *$ \\
\hline & $(0,117)$ & $(0,117)$ & $(0,117)$ & $(0,116)$ & $(0,117)$ & $(0,116)$ \\
\hline \multirow[t]{2}{*}{$\mathrm{DFC}$} & $-0,001$ & $-0,001$ & $-0,001$ & $-0,001$ & $-0,001$ & $-0,001 * *$ \\
\hline & $(0,001)$ & $(0,001)$ & $(0,001)$ & $(0,001)$ & $(0,001)$ & $(0,001)$ \\
\hline \multirow[t]{2}{*}{ RxDFC } & $0,003 * *$ & $0,003 * *$ & $0,003 * *$ & $0,003 * *$ & $0,003^{* *}$ & $0,003 * *$ \\
\hline & $(0,001)$ & $(0,001)$ & $(0,001)$ & $(0,001)$ & $(0,001)$ & $(0,001)$ \\
\hline \multirow[t]{2}{*}{ DRxDFC } & 0,001 & 0,001 & 0,001 & $0,002 *$ & $0,002 *$ & $0,002 *$ \\
\hline & $(0,001)$ & $(0,001)$ & $(0,001)$ & $(0,001)$ & $(0,001)$ & $(0,001)$ \\
\hline \multirow[t]{2}{*}{ DRxRxDFC } & $-0,006 * * *$ & $-0,006^{* * *}$ & $-0,006 * * *$ & $-0,006^{* * *}$ & $-0,007 * * *$ & $-0,007 * * *$ \\
\hline & $(0,002)$ & $(0,002)$ & $(0,002)$ & $(0,002)$ & $(0,002)$ & $(0,002)$ \\
\hline \multirow[t]{2}{*}{ Constant } & $0,082 * * *$ & $0,088 * * *$ & 0,082 & $0,231 * * *$ & $0,099 * * *$ & $0,268^{* * *}$ \\
\hline & $(0,029)$ & $(0,030)$ & $(0,056)$ & $(0,059)$ & $(0,032)$ & $(0,074)$ \\
\hline IFRS & & SI & & & & SI \\
\hline CONTROL & & & SI & & & SI \\
\hline SECTOR & & & & SI & & SI \\
\hline AÑO & & & & & SI & SI \\
\hline Observations & 529 & 529 & 525 & 529 & 529 & 525 \\
\hline R-squared & 0,195 & 0,196 & 0,209 & 0,234 & 0,224 & 0,277 \\
\hline
\end{tabular}

Coeficientes estimados (estadístico-t) de la Ecuación (2) para las empresas de la muestra.

$X_{i t}=\alpha+\beta_{1} * R_{i t}+\beta_{2} * D R_{i t}+\beta_{3} * D R_{i t} * R_{i t}+\beta_{4} * D F C_{i t}+\beta_{5} * R_{i t} * D F C{ }_{i t}$

$+\beta_{6} * D R_{i t} * D F C_{i t}+\beta_{7} * D R_{i t} * R * D F C_{i t}$.

*** indica un nivel de significación para un contraste de dos colas inferior al $1 \%$,

$* *$ inferior al $5 \% \mathrm{y}$

* inferior al $10 \%$.

Error estándar en paréntesis. 
Como se puede apreciar, en todas las columnas se tiene que el coeficiente de la variable $D R_{i t} * R_{i t}\left(\beta_{3}\right)$ es positivo y estadísticamente significativo, lo que evidencia que las utilidades contables son más sensibles a las malas noticias $o$, es decir, existe reconocimiento asimétrico de las utilidades. También se tiene que el coeficiente del término $D R_{i t} * R_{i t} * D F C_{i t}\left(\beta_{7}\right)$ es negativo y estadísticamente significativo, lo que indica que este reconocimiento asimétrico de las utilidades disminuye a medida que aumenta la participación de la propiedad que tiene el accionista mayoritario, lo que es consistente con la hipótesis 1 que se planteó en un comienzo.

Cada una de las columnas de cada tabla de resultados difiere de acuerdo con la combinación y uso de las distintas variables de control empleadas en el modelo.

Por su parte, en la tabla 2 se muestran los resultados de las estimaciones para la ecuación (3), también utilizando distintas interacciones de las variables de control, en donde se analiza el efecto de la concentración de propiedad que tiene el accionista mayoritario de la empresa sobre el conservadurismo contable, pero esta vez desde el punto de vista de los derechos de voto que tiene.

En este caso los resultados arrojan el mismo comportamiento de las variables que en la Ecuación (2), pero esta vez los resultados solo son estadísticamente significativos para el término $D R_{i t} * R_{i t} *$ $D V_{i t}\left(\beta_{7}\right)$.

Por último, en la tabla 3 se analiza la ecuación (4), donde se tiene que el efecto asimétrico marginal que tienen las malas noticias dependiente de si existe o no diferencia entre los derechos de flujos de caja y derechos de voto, $\beta_{11}\left(D R_{i t} * R_{i t} * D V D F C_{i t}\right)$, es negativo, pero no es estadísticamente significativo. Por tanto, no es posible determinar que si existen estructuras de control piramidales en la empresa, se tendrá una disminución en el conservadurismo contable, por lo que no hay consistencia con la hipótesis 2 que se planteó anteriormente. 


\section{Tabla 2}

\section{Conservadurismo contable $y$ derechos de flujo de caja}

\begin{tabular}{|c|c|c|c|c|c|c|}
\hline \multirow[b]{2}{*}{ VARIABLES } & (1) & (2) & (3) & (4) & (5) & (6) \\
\hline & $\mathrm{X}$ & $\mathrm{X}$ & $\mathrm{X}$ & $\mathrm{X}$ & $X$ & $\mathrm{X}$ \\
\hline \multirow[t]{2}{*}{$\mathrm{R}$} & 0,069 & 0,071 & 0,068 & 0,054 & 0,057 & 0,035 \\
\hline & $(0,059)$ & $(0,060)$ & $(0,059)$ & $(0,059)$ & $(0,059)$ & $(0,058)$ \\
\hline \multirow[t]{2}{*}{ DR } & $-0,036$ & $-0,037$ & $-0,030$ & $-0,047$ & $-0,047$ & $-0,049$ \\
\hline & $(0,048)$ & $(0,048)$ & $(0,048)$ & $(0,048)$ & $(0,048)$ & $(0,048)$ \\
\hline \multirow[t]{2}{*}{ DRxR } & 0,204 & 0,208 & 0,194 & 0,213 & $0,266^{*}$ & $0,270^{* *}$ \\
\hline & $(0,138)$ & $(0,138)$ & $(0,137)$ & $(0,136)$ & $(0,137)$ & $(0,135)$ \\
\hline \multirow[t]{2}{*}{ DV } & $-0,000$ & $-0,000$ & $-0,000$ & $-0,000$ & $-0,000$ & $-0,001$ \\
\hline & $(0,001)$ & $(0,001)$ & $(0,001)$ & $(0,001)$ & $(0,001)$ & $(0,001)$ \\
\hline \multirow[t]{2}{*}{$\mathrm{RxDV}$} & 0,001 & 0,001 & 0,001 & 0,002 & 0,002 & 0,002 \\
\hline & $(0,001)$ & $(0,001)$ & $(0,001)$ & $(0,001)$ & $(0,001)$ & $(0,001)$ \\
\hline \multirow[t]{2}{*}{ DRxDV } & 0,000 & 0,000 & 0,000 & 0,001 & 0,001 & 0,001 \\
\hline & $(0,001)$ & $(0,001)$ & $(0,001)$ & $(0,001)$ & $(0,001)$ & $(0,001)$ \\
\hline \multirow[t]{2}{*}{ DRxRxDV } & $-0,005^{* *}$ & $-0,005^{* *}$ & $-0,005 * *$ & $-0,006^{* *}$ & $-0,006 * * *$ & $-0,006 * * *$ \\
\hline & $(0,002)$ & $(0,002)$ & $(0,002)$ & $(0,002)$ & $(0,002)$ & $(0,002)$ \\
\hline \multirow{2}{*}{ Constant } & 0,051 & $0,057^{*}$ & 0,061 & $0,209 * * *$ & $0,073 * *$ & $0,260 * * *$ \\
\hline & $(0,032)$ & $(0,033)$ & $(0,058)$ & $(0,062)$ & $(0,035)$ & $(0,076)$ \\
\hline IFRS & & SI & & & & SI \\
\hline CONTROL & & & SI & & & SI \\
\hline SECTOR & & & & SI & & SI \\
\hline AÑO & & & & & SI & SI \\
\hline Observations & 529 & 529 & 525 & 529 & 529 & 525 \\
\hline R-squared & 0,187 & 0,187 & 0,200 & 0,226 & 0,216 & 0,268 \\
\hline
\end{tabular}

Coeficientes estimados (estadístico-t) de la Ecuación (3) para las empresas de la muestra. $X_{i t}=\alpha+\beta_{1} * R_{i t}+\beta_{2} * D R_{i t}+\beta_{3} * D R_{i t} * R_{i t}+\beta_{4} * D V_{i t}+\beta_{5} * R_{i t} * D V_{i t}$ $+\beta_{6} * D R_{i t} * D V_{i t}+\beta_{7} * D R_{i t} * R * D V_{i t}$.

$* * *$ indica un nivel de significación para un contraste de dos colas inferior al $1 \%$, $* *$ inferior al $5 \%$ y

$*$ inferior al $10 \%$.

Error estándar en paréntesis. 
Tabla 3

Conservadurismo contable y separación $D V-D F C$

\begin{tabular}{|c|c|c|c|c|c|c|}
\hline & (1) & (2) & (3) & (4) & (5) & (6) \\
\hline VARIABLES & $\mathrm{X}$ & $\mathrm{X}$ & $\mathrm{X}$ & $\mathrm{X}$ & $\mathrm{X}$ & $\mathrm{X}$ \\
\hline \multirow[t]{2}{*}{$\mathrm{R}$} & 0,035 & 0,036 & 0,039 & 0,024 & 0,021 & 0,011 \\
\hline & $(0,066)$ & $(0,066)$ & $(0,066)$ & $(0,066)$ & $(0,066)$ & $(0,065)$ \\
\hline \multirow[t]{2}{*}{ DR } & $-0,074$ & $-0,075$ & $-0,066$ & $-0,080$ & $-0,082$ & $-0,078$ \\
\hline & $(0,055)$ & $(0,055)$ & $(0,055)$ & $(0,054)$ & $(0,054)$ & $(0,054)$ \\
\hline \multirow[t]{2}{*}{ DRxR } & $0,230^{*}$ & $0,233^{*}$ & 0,199 & $0,247^{*}$ & $0,296 * *$ & $0,288^{* *}$ \\
\hline & $(0,137)$ & $(0,137)$ & $(0,137)$ & $(0,135)$ & $(0,137)$ & $(0,135)$ \\
\hline \multirow[t]{2}{*}{ DFC } & $-0,000$ & $-0,000$ & $-0,000$ & $-0,001$ & $-0,001$ & $-0,001$ \\
\hline & $(0,001)$ & $(0,001)$ & $(0,001)$ & $(0,001)$ & $(0,001)$ & $(0,001)$ \\
\hline \multirow[t]{2}{*}{ RxDFC } & 0,002 & 0,002 & 0,002 & 0,002 & $0,003^{*}$ & $0,003 * *$ \\
\hline & $(0,002)$ & $(0,002)$ & $(0,002)$ & $(0,002)$ & $(0,002)$ & $(0,002)$ \\
\hline \multirow[t]{2}{*}{ DRxDFC } & 0,001 & 0,001 & 0,001 & 0,002 & 0,002 & $0,002^{*}$ \\
\hline & $(0,001)$ & $(0,001)$ & $(0,001)$ & $(0,001)$ & $(0,001)$ & $(0,001)$ \\
\hline \multirow[t]{2}{*}{ DRxRxDFC } & $-0,006^{* * *}$ & $-0,006 * * *$ & $-0,006 * *$ & $-0,006^{* * *}$ & $-0,007 * * *$ & $-0,007 * * *$ \\
\hline & $(0,002)$ & $(0,002)$ & $(0,002)$ & $(0,002)$ & $(0,002)$ & $(0,002)$ \\
\hline \multirow[t]{2}{*}{ DVDFC } & 0,025 & 0,026 & 0,037 & 0,024 & 0,017 & 0,027 \\
\hline & $(0,032)$ & $(0,032)$ & $(0,032)$ & $(0,031)$ & $(0,031)$ & $(0,031)$ \\
\hline \multirow[t]{2}{*}{ RxDVDFC } & $-0,055$ & $-0,056$ & $-0,062$ & $-0,048$ & $-0,040$ & $-0,034$ \\
\hline & $(0,050)$ & $(0,050)$ & $(0,050)$ & $(0,049)$ & $(0,049)$ & $(0,049)$ \\
\hline \multirow[t]{2}{*}{ DRxDVDFC } & $-0,008$ & $-0,008$ & $-0,007$ & $-0,010$ & $-0,000$ & $-0,003$ \\
\hline & $(0,046)$ & $(0,046)$ & $(0,046)$ & $(0,045)$ & $(0,045)$ & $(0,045)$ \\
\hline \multirow[t]{2}{*}{ DRxRxDVDFC } & $-0,052$ & $-0,051$ & $-0,003$ & $-0,084$ & $-0,078$ & $-0,070$ \\
\hline & $(0,115)$ & $(0,115)$ & $(0,116)$ & $(0,115)$ & $(0,114)$ & $(0,114)$ \\
\hline \multirow[t]{2}{*}{ Constant } & $0,065^{*}$ & $0,071^{*}$ & 0,065 & $0,218 * * *$ & $0,087^{* *}$ & $0,259 * * *$ \\
\hline & $(0,039)$ & $(0,040)$ & $(0,060)$ & $(0,064)$ & $(0,041)$ & $(0,077)$ \\
\hline IFRS & & SI & & & & SI \\
\hline CONTROL & & & SI & & & SI \\
\hline SECTOR & & & & SI & & SI \\
\hline AÑO & & & & & SI & SI \\
\hline Observations & 529 & 529 & 525 & 529 & 529 & 525 \\
\hline R-squared & 0,203 & 0,204 & 0,218 & 0,243 & 0,232 & 0,285 \\
\hline
\end{tabular}

Coeficientes estimados (estadístico-t) de la Ecuación (4) para las empresas de la muestra.

$X_{i t}=\alpha+\beta_{1} * R_{i t}+\beta_{2} * D R_{i t}+\beta_{3} * D R_{i t} * R_{i t}+\beta_{4} * D F C_{i t}+\beta_{5} * R_{i t} * D F C_{i t}$

$$
\begin{aligned}
& +\beta_{6} * D R_{i t} * D F C_{i t}+\beta_{7} * D R_{i t} * R * D F C_{i t}+\beta_{8} * D_{V D F C} i t+\beta_{9} * R * D V D F C_{i t} \\
& +\beta_{10} * D R_{i t} * D V D F C_{i t}+\beta_{11} * D R_{i t} * R_{i t} * D V D F C_{i t}
\end{aligned}
$$

*** indica un nivel de significación para un contraste de dos colas inferior al $1 \%$,

** inferior al $5 \% \mathrm{y}$

*inferior al $10 \%$.

Error estándar en paréntesis 


\section{Conclusiones}

Uno de los principales elementos que se necesita en un mercado financiero para su correcto funcionamiento es el flujo de información, y que este garantice que sea oportuna y fidedigna. Entre las distintas fuentes de información se encuentra aquella contenida en los estados financieros y, en especial, en las utilidades contables, la que resulta relevante debido a sus implicaciones en la medición del desempeño y en la valoración de las empresas.

En este sentido, las propiedades de las utilidades contables resultan un factor clave en el proceso de toma de decisiones. El grado de conservadurismo de las utilidades contables, entendida como la tendencia a reconocer asimétricamente las buenas y malas noticias económicas en las utilidades contables con el tiempo, puede ser un factor relevante al momento de velar por información oportuna y fidedigna, atenuando ciertos problemas de información asimétrica.

Este trabajo tiene como principal objetivo aportar a la literatura que trata respecto del conservadurismo contable, entendiendo además qué tan conservador es el mercado financiero chileno y analizar los efectos que tiene la estructura de propiedad en el conservadurismo contable.

Los resultados indican que efectivamente en el mercado chileno, como es señalado en literatura previa, se encuentra que la concentración de propiedad influye negativamente en el nivel de conservadurismo contable, es decir, a medida que aumenta la participación de propiedad que tiene el accionista controlador, disminuye la tendencia a reconocer anticipadamente en las utilidades contables las malas noticia versus las buenas noticias.

No obstante, no es posible determinar que en empresas con estructuras de propiedad piramidales disminuye el nivel de conservadurismo contable, por lo que no hay consistencia con la hipótesis 2 planteada en el documento. 
Por último, considero que es necesario reforzar algunos de estos resultados (primera hipótesis) e intentar encontrar otros que apoyen la literatura previa (segunda hipótesis) utilizando una muestra más amplia, que considere un rango de tiempo mayor, que permita utilizar otra metodología, por ejemplo el Método Generalizado de Momentos (GMM) y que así haga obtener resultados más representativos del mercado financiero local. 


\section{Referencias}

Almeida, H. and D. Wolfenzon (2006), "A Theory of Pyramidal Ownership and Family Business Groups”, The Journal of Finance, Vol. LXI, Nº 6.

BALL, R. (2001), "Infrastructure requirements for an economically efficient system of public financial reporting and disclosure", Brookings-Wharton Papers on Financial Services, Vol. 2001(1), pp. 127-169.

BALL, R. and L. SHIVAKUMAR (2006), "The role of accruals in asymmetrically timely gain and loss recognition", Journal of Accounting Research, Vol. 44(2), pp. 207-242.

BASU, S. (1997), "The conservatism principle and the asymmetric timeliness of earnings", Journal of Accounting and Economics, Vol. 24(1), pp. 3-37.

BAXter, P. and J. CotTer (2009), "Audit committees and earnings quality", Accounting \& Finance, Vol. 49(2), pp. 267-290.

BEBCHUK, L. A. (1999), "A rent-protection theory of corporate ownership and control”, National Bureau of Economic Research, ( ${ }^{\circ}$ w7203).

Bona-SÁnchez, C., J. PÉrez-Alemán and D.J. SAntana-Martín (2011), "Ultimate ownership and earnings conservatism", European Accounting Review, Vol. 20(1), pp. 57-80.

Bushman, R.M. and J.D. Piotroski (2006), "Financial reporting incentives for conservative accounting: The influence of legal and political institutions", Journal of Accounting and Economics, Vol. 42(1-2), pp. 107-148.

DARgenidou, C., S. MCLeAY and I. RAONIC (2007), "Ownership, investor protection and earnings expectations", Journal of Business Finance \& Accounting, Vol. 34(1-2), pp. 247-268.

Cestone, G. and C. Fumagalli (2005), "The strategic impact of resource flexibility in business groups", RAND Journal of Economics, Vol. 36(1), pp. 193-214.

Claessens, S., S. DJAnkov and L.H. LANG (2000), "The separation of ownership and control in East Asian corporations", Journal of financial Economics, Vol. 58(1-2), pp. 81-112. 
Desai, M. A., C.F. Foley and J.R. HinES (2004), “A multinational perspective on capital structure choice and internal capital markets", The Journal of Finance, Vol. 59(6), pp. 2451-2487.

DU, J. and Y. DAI (2005), "Ultimate corporate ownership structures and capital structures: Evidence from East Asian economies", Corporate Governance: An International Review, Vol. 13(1), pp. 60-71.

FACCIO, M., L.H. LANG and L. Young (2001), "Dividends and expropriation", American Economic Review, Vol. 91(1), pp. 54-78.

FACCIO, M. and L.H. LANG (2002), "The ultimate ownership of Western European corporations", Journal of Financial Economics, Vol. 65(3), pp. 365-395.

FrANCIS, J., K. SCHIPPER and L. VINCENT (2005), "Earnings and dividend informativeness when cash flow rights are separated from voting rights", Journal of Accounting and Economics, Vol. 39(2), pp. 329-360.

Givoly, D., C.K. HAYN and A. NATARAJAN (2007), "Measuring reporting conservatism", The Accounting Review, Vol. 82(1), pp. 65-106.

JARA, M. and J. ARIAS (2012), "The effect of mandatory IFRS adoption on accounting conservatism of reported earnings: evidence from Chilean firms", Academia Revista Latinoamericana de Administración, 26(1), pp. 139-169.

Jensen, M. C. and W.H. MeCKLING (1976), "Theory of the firm: Managerial behavior, agency costs and ownership structure", Journal of Fnancial Economics, Vol. 3(4), pp. 305-360.

KLAPPER, L. F. and I. LOVE (2004), “Corporate governance, investor protection, and performance in emerging markets", Journal of Corporate Finance, Vol. 10(5), pp. 703-728.

Kothari, S. P., K. RAMANNA and D.J. SKINNER (2010), "Implications for GAAP from an analysis of positive research in accounting", Journal of Accounting and Economics, Vol. 50(2-3), pp. 246-286.

LA Porta, R., F. LopeZ-De-Silanes and A. Shleifer (1999), "Corporate ownership around the world", The journal of Finance, Vol. 54(2), pp. 471-517. 
LA FOND, R. (2005), "The influence of ownership structure on earnings conservatism and the informativeness of stock prices: An international comparison", Sloan School of Management, Working Paper.

LA FOND, R. and S. ROYCHOWDHURY (2008), "Managerial ownership and accounting conservatism", Journal of Accounting Research, Vol. 46(1), pp. 101-135.

Peek, E., R. CuiJpers and W. BuiJink (2010), “Creditors' and shareholders' reporting demands in public versus private firms: Evidence from Europe", Contemporary Accounting Research, Vol. 27(1), pp. 49-91.

ROYCHOWDHURY, S. and R.L. WATTS (2007), "Asymmetric timeliness of earnings, market-to-book and conservatism in financial reporting", Journal of Accounting and Economics, Vol. 44(1-2), pp. 2-31.

VillalongA, B. and R. Amit (2006), "How do family ownership, control and management affect firm value?", Journal of Financial Economics, Vol. 80(2), pp. 385-417.

WATTS, R.L. (2003a), “Conservatism in accounting part I: Explanations and implications”, Accounting Horizons, Vol. 17(3), pp. 207-221.

WATTS, R.L. (2003b), “Conservatism in accounting part II: Evidence and research opportunities”, Accounting Horizons, Vol. 17(4), pp. 287-301. 
